\title{
A RELATION BETWEEN RIESZ AND RIEMANN SUMMABILITY
}

\author{
H. BURKILL AND G. M. PETERSEN
}

The Cesàro and the Riemann methods of summation are both intimately connected with the theories of Fourier and trigonometric series. It is therefore natural that the relation between these methods should have been investigated in some detail. For instance Verblunsky [2] has proved that, when $k$ is a positive integer, summability $(C, k-\delta)$ implies summability $(R, k+1)$; and Kuttner [1] has proved that, for $k=1,2$, summability $(R, k)$ implies summability $(C, k+\delta)$.

Riesz's typical means generalise Cesaro summability and there is a corresponding generalisation for Riemann summability. Since both these methods are appropriate for dealing with almost periodic functions, we wish to establish a connection between them.

The notation we shall use is the following.

Riesz's typical means. If $k>0,0=\lambda_{0}<\lambda_{1}<\lambda_{2}<\cdots$ and

$$
\sum_{\lambda_{n}<\omega}\left(1-\frac{\lambda_{n}}{\omega}\right)^{k} u_{n} \rightarrow s
$$

as $\omega \rightarrow \infty$, then $\sum u_{n}$ is said to be summable $\left(R, \lambda_{n}, k\right)$ to $s$.

Riemann summability. If $k$ is a positive rational number with odd denominator, $0<\lambda_{1}<\lambda_{2}<\cdots$ and

$$
u_{0}+\sum_{n=1}^{\infty}\left(\frac{\sin \lambda_{n} h}{\lambda_{n} h}\right)^{k} u_{n} \rightarrow s
$$

as $h \rightarrow 0$, then $\sum u_{n}$ is said to be summable $\left(R, k, \lambda_{n}\right)$ to $s$.

The object of this note is to prove the following result.

THEOREM. Suppose $\lambda_{0}=0,0<p \leqq \lambda_{n+1}-\lambda_{n} \leqq q$ for all $n$ and $\sum_{n=0}^{\infty} u_{n}$ is summable $\left(R, \lambda_{n}, 1\right)$ to $t$. Then $\sum_{n=0}^{\infty} u_{n}$ is also summable $\left(R, k, \lambda_{n}\right)$ to $t$ when $k>2$ (and $k$ is a rational number with odd denominator).

Before proceeding with the proof we might mention that, although our result is new even when $\left\{\lambda_{n}\right\}=\{n\}$, from the point of view of applications it would be more desirable to proceed from a nonintegral Riesz mean to Riemann summability of an integral order. However this problem appears to be a very formidable bne because of the diffculty of dealing with nonintegral Riesz means.

Received by the editors April 22, 1960 and, in revised form, June 20, 1960. 
ProOF OF THE THEOREM. For $n \geqq 1$ we put

$$
t_{n}=\sum_{r=0}^{n}\left(1-\frac{\lambda_{r}}{\lambda_{n}}\right) u_{r}
$$

so that the hypothesis of the theorem implies that $t_{n} \rightarrow t$ as $n \rightarrow \infty$. We have

$$
\lambda_{n} t_{n}=\lambda_{n} s_{n}-\sum_{r=0}^{n} \lambda_{r} u_{r}
$$

where $s_{n}=u_{0}+u_{1}+\cdots+u_{n}$, and so

$$
\lambda_{n+1} t_{n+1}-\lambda_{n} t_{n}=\lambda_{n+1} s_{n+1}-\lambda_{n} s_{n}-\lambda_{n+1} u_{n+1}=\left(\lambda_{n+1}-\lambda_{n}\right) s_{n} .
$$

It follows that, for $n \geqq 2$,

$$
u_{n}=\frac{\lambda_{n+1} t_{n+1}-\lambda_{n} t_{n}}{\lambda_{n+1}-\lambda_{n}}-\frac{\lambda_{n} t_{n}-\lambda_{n-1} t_{n-1}}{\lambda_{n}-\lambda_{n-1}}
$$

and, since $\lambda_{0}=0,(1)$ is easily seen to hold for $n=1$ also, whatever value is assigned to $t_{0}$.

Taking a suitable $k>2$ we define $\tau_{h}$, for $h>0$, by

$$
\tau_{h}=u_{0}+\sum_{n=1}^{\infty}\left(\frac{\sin \lambda_{n} h}{\lambda_{n} h}\right)^{k} u_{n}
$$

the series being convergent since $\lambda_{n+1}-\lambda_{n} \geqq p$ and so $u_{n}=o\left(\lambda_{n}\right)$. We put

$$
c(x)=\left\{\begin{array}{cl}
1, & \text { for } x=0, \\
\left(\frac{\sin x}{x}\right)^{k}, & \text { for } x \neq 0 .
\end{array}\right.
$$

Then, by (1) and since also $u_{0}=t_{1}$,

$$
\tau_{h}=c\left(\lambda_{0} h\right) t_{1}+\sum_{n=1}^{\infty} c\left(\lambda_{n} h\right)\left(\frac{\lambda_{n+1} t_{n+1}-\lambda_{n} t_{n}}{\lambda_{n+1}-\lambda_{n}}-\frac{\lambda_{n} t_{n}-\lambda_{n-1} t_{n-1}}{\lambda_{n}-\lambda_{n-1}}\right) .
$$

Now $t_{n}$ is bounded, $c\left(\lambda_{n} h\right)=O\left(1 / n^{k}\right)$ and $\lambda_{n} /\left(\lambda_{r+1}-\lambda_{r}\right)=O(n)$. Hence the series for $\tau_{h}$ may be written

$$
\begin{aligned}
\tau_{h} & =\sum_{n=1}^{\infty}\left(\frac{c\left(\lambda_{n+1} h\right)-c\left(\lambda_{n} h\right)}{\lambda_{n+1}-\lambda_{n}}-\frac{c\left(\lambda_{n} h\right)-c\left(\lambda_{n-1} h\right)}{\lambda_{n}-\lambda_{n-1}}\right) \lambda_{n} t_{n} \\
& =\sum_{n=1}^{\infty} \alpha_{h, n} t_{n} .
\end{aligned}
$$


We wish to prove that this transformation is regular, i.e. that $\tau_{h} \rightarrow t$ as $h \rightarrow 0$. This is so if (and only if) the three Toeplitz conditions are satisfied, namely

(i) $\alpha_{h, n} \rightarrow 0$ as $h \rightarrow 0$, for all $n$,

(ii) $\sum_{n=1}^{\infty}\left|\alpha_{h, n}\right|$ is uniformly bounded for all $h>0$,

(iii) $\sum_{n=1}^{\infty} \alpha_{h, n} \rightarrow 1$ as $h \rightarrow 0$.

The first and the third condition are easily shown to be satisfied. In the first place the continuity of $c(x)$ shows that $\alpha_{h, n} \rightarrow 0$ as $h \rightarrow 0$. Also

$$
\sum_{n=1}^{\infty} \alpha_{h, n}=1
$$

for all $h>0$. This is either proved directly or by taking $u_{0}=1, u_{n}=0$ for $n \geqq 1$ so that $t_{n}=1$ for all $n$ and $\tau_{h}=1$ for all $h>0$.

We still have to prove (ii). We have

$$
\begin{aligned}
\alpha_{h, n} & =\left(\frac{c\left(\lambda_{n+1} h\right)-c\left(\lambda_{n} h\right)}{\lambda_{n+1} h-\lambda_{n} h}-\frac{c\left(\lambda_{n} h\right)-c\left(\lambda_{n-1} h\right)}{\lambda_{n} h-\lambda_{n-1} h}\right) \lambda_{n} h \\
& =\left\{c^{\prime}\left(\eta_{n}\right)-c^{\prime}\left(\xi_{n}\right)\right\} \lambda_{n} h \\
& =\left(\eta_{n}-\xi_{n}\right) c^{\prime \prime}\left(x_{n}\right) \lambda_{n} h,
\end{aligned}
$$

where $\lambda_{n-1} h<\xi_{n}<\lambda_{n} h<\eta_{n}<\lambda_{n+1} h$ and $\xi_{n}<x_{n}<\eta_{n}$. Now

$$
\begin{aligned}
c^{\prime \prime}(x)=k(k-1) & \left(\frac{\sin x}{x}\right)^{k-2}\left(\frac{x \cos x-\sin x}{x^{2}}\right)^{2} \\
& +k\left(\frac{\sin x}{x}\right)^{k-1}\left(\frac{-x^{2} \sin x-2 x \cos x+2 \sin x}{x^{3}}\right)
\end{aligned}
$$

and so

$$
\left|c^{\prime \prime}(x)\right|< \begin{cases}A & \text { if } 0<x<1, \\ A / x^{k} & \text { if } x \geqq 1,\end{cases}
$$

where $A$ is a constant.

Let $N$ be the integer such that

$$
\lambda_{N} h<1 \leqq \lambda_{N+1} h .
$$

For $n \leqq N$,

$$
\left|\alpha_{h, n}\right|<\left(\eta_{n}-\xi_{n}\right) A \lambda_{n} h<2 q h A \lambda_{n} h<2 A q^{2} n h^{2}
$$

and so 


$$
\begin{aligned}
\sum_{n=1}^{N}\left|\alpha_{h, n}\right| & <2 A q^{2} h^{2} \sum_{n=1}^{N} n=A q^{2} h^{2} N(N+1)<B h^{2} N^{2} \\
& \leqq B h^{2}\left(\lambda_{N} / p\right)^{2}<B / p^{2}=K
\end{aligned}
$$

say.

For $n>N$,

$$
\left|\alpha_{h, n}\right|<\left(\eta_{n}-\xi_{n}\right) \frac{A}{x_{n}^{k}} \lambda_{n} h<\frac{2 A q^{2} n h^{2}}{\left(\lambda_{n-1} h\right)^{k}} \leqq \frac{2 A q^{2}}{\left(\frac{1}{2} p\right)^{k} h^{k-2} n^{k-1}}
$$

and so

$$
\begin{aligned}
& \sum_{n=N+1}^{\infty}\left|\alpha_{h, n}\right|<\frac{2 A q^{2}}{\left(\frac{1}{2} p\right)^{k} h^{k-2}} \sum_{n=N+1}^{\infty} \frac{1}{n^{k-1}}<\frac{C}{h^{k-2}(N+1)^{k-2}} \\
& <\frac{C q^{k-2}}{\left(h \lambda_{N+1}\right)^{k-2}} \leqq C q^{k-2}=L,
\end{aligned}
$$

say. Hence, for all $h>0$,

$$
\sum_{n=1}^{\infty}\left|\alpha_{h, n}\right|<K+L
$$

and the theorem is therefore proved.

We wish to express our thanks to the Sandia Corporation of Albuquerque, New Mexico, which financed a project of which this paper is a part.

\section{REFERENCES}

1. B. Kuttner, The relation between Riemann and Cesdro summability, Proc. London Math. Soc. (2) vol. 38 (1935) pp. 273-283.

2. S. Verblunsky, The relation between Riemann's method of summation and Cesdro's, Proc. Cambridge Philos. Soc. vol. 26 (1930) pp. 34-42.

University of Sheffield, England and

University College of Swansea, Wales 